\title{
Nonlinear optical properties of photoresists for projection lithography
}

\author{
Anthony S. Kewitsch ${ }^{\mathrm{a})}$ and Amnon Yariv \\ Department of Applied Physics, California Institute of Technology 128-95, Pasadena, California 91125
}

(Received 8 September 1995; accepted for publication 14 November 1995)

\begin{abstract}
Optical beams are self-focused and self-trapped upon initiating crosslinking in photoresists. This nonlinear optical phenomenon is apparent only for low average optical intensities and produces index of refraction changes as large as 0.04 . We propose using the self-focusing and self-trapping phenomenon in projection photolithography to enhance the resolution and depth of focus. (C) 1996 American Institute of Physics. [S0003-6951(96)02003-0]
\end{abstract}

The primary obstacle to the continued increase in integrated circuit density is the limitation on the resolution and depth of focus in the photolithography stage. The smallest feature size $x_{\text {min }}$ that can be projected by a coherent imaging system is $x_{\min }=\lambda /(2 \mathrm{NA})$, and the depth of focus (DOF) is $\mathrm{DOF}=\lambda /\left[2(\mathrm{NA})^{2}\right]$, where $\lambda$ is the wavelength of the illumination and NA is the numerical aperture. The most direct route to attaining smaller feature sizes is to reduce the wavelength from the $i$-line standard of today $(365 \mathrm{~nm})$ to excimer laser wavelengths (248 or $193 \mathrm{~nm}$ ). The NA is typically 0.5 , so the feature size is on the order of the exposure wavelength. State of the art semiconductor fabrication facilities in the year 2005 are forecasted to use a $0.1 \mu \mathrm{m}$ process, at which point the resolution and depth of focus constraints of optical lithography become severe. Therefore, techniques to enhance the performance of optical lithography are of particular technological and economic significance.

As the fundamental limits of optical lithography are approached, the nonlinear optical properties of the photoresist become increasingly important. This nonlinearity arises from the change in the complex index of refraction of the photoresist under UV exposure. A common nonlinear optical phenomenon is the light-induced change in the imaginary part of the index of refraction (i.e., absorption) upon exposure. For instance, contrast enhancement layers (CEL) composed of a photobleachable dye are often overcoated on a photoresist ${ }^{1-3}$ to introduce exposure thresholding. This improves the contrast of the projected image. In this letter we instead exploit the permanent optical nonlinearity in the real part of the complex index of refraction. That is, when a negative photoresist is illuminated with low intensity UV radiation, the exposed regions crosslink to form a solid with a real index of refraction typically 0.01 to 0.1 larger than the liquid. This index change leads to self-focusing, in which light induces a lens, and self-trapping, in which light induces an optical waveguide. $^{4}$

The propagation of light within the photoresist is described analytically by the scalar, three-dimensional, paraxial wave equation for the optical electric field amplitude $E$ in an inhomogeneous medium:

\footnotetext{
${ }^{\text {a)} P r e s e n t ~ a d d r e s s: ~ A r r o y o ~ O p t i c s, ~ I n c . ~} 1646$ 17th St., Santa Monica, CA 90404.
}

$$
\begin{gathered}
2 i k n_{0} \frac{\partial E}{\partial z}+\frac{\partial^{2} E}{\partial x^{2}}+\frac{\partial^{2} E}{\partial y^{2}}+k^{2}\left[\left(n^{\prime}+i n^{\prime \prime}\right)^{2}\right. \\
\left.-\left(n_{0}^{\prime}+i n_{0}^{\prime \prime}\right)^{2}\right] E=0,
\end{gathered}
$$

where $n$ and $n_{0}$ are the spatially modulated and unperturbed indices of refraction, respectively, $k=2 \pi / \lambda$ is the wave vector for a paraxial beam propagating along the $z$ direction and $\lambda$ is the wavelength in vacuum. The medium is nonlinear because the index of refraction and absorption described by $n$ depend on optical exposure (i.e., the time integral of the square modulus of the optical field $E$ ), time and position $x, y, z$.

A density change upon light-induced crosslinking is the dominant effect contributing to the index change in photopolymers. The largest index changes are expected to be less than or equal to $0.15,{ }^{5}$ which is two orders of magnitude larger than that of traditional nonlinear optical phenomena such as the Kerr or photorefractive effects. However, unlike the Kerr effect, the index response upon illumination is not instantaneous. An index change develops on the time scale of $1 \mathrm{~ms}$ to $1 \mathrm{~s}$, the lifetime of the photogenerated radicals. We measure this evolution of the index of refraction of the photopolymer during crosslinking with an Abbe refractometer at $589.3 \mathrm{~nm}$ (Fig. 1).

Some qualitative predictions regarding the evolution of the light-induced lens and waveguide can be deduced from Fig. 1 and the nonlinear wave equation. For low exposures $\left(<7 \mathrm{~mJ} \mathrm{~cm}^{-2}\right)$, the induction period and the delay in the material response impede self-focusing. Polymerization is initiated first in regions of intense illumination, so the lateral dimension of the lens is smaller than that of the optical

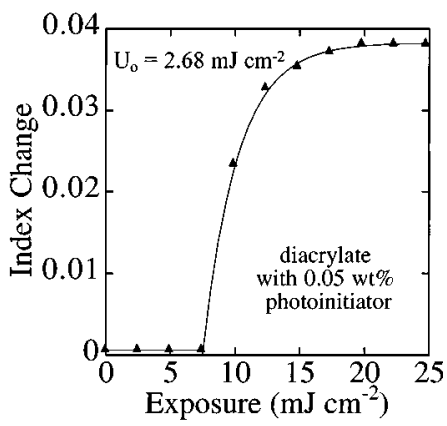

FIG. 1. Measured evolution of the index of refraction upon optical exposure at $325 \mathrm{~nm}$. 


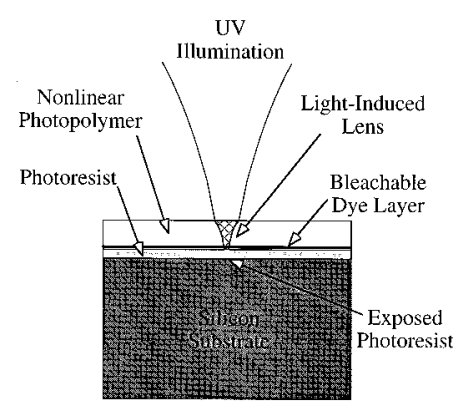

(a)

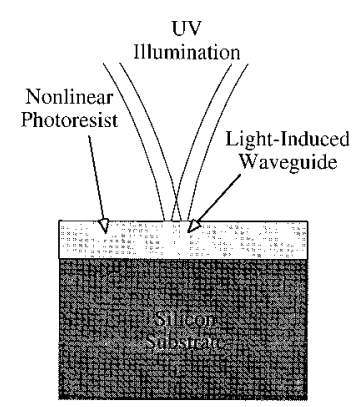

(b)
FIG. 2. (a) Resolution enhancement layer overcoated on standard photoresist reduces the linewidth before reaching underlying resist. (b) Nonlinear photoresist exhibiting self-trapping allows the patterning of thick resist layers.

beam. As the exposure increases, the index increases linearly with exposure and the index distribution becomes a faithful replica of the transverse intensity profile. Self-focusing and trapping is strongest during this stage. For large exposures $\left(>15 \mathrm{~mJ} \mathrm{~cm}^{-2}\right)$, the index change saturates and self-focusing decreases. Of course, for very large exposures the entire liquid will polymerize because of the scatter of light and the diffusion of free radicals beyond the illuminated region.

A unique condition for observing self-focusing and selftrapping in photopolymers is that the average intensity must lie below an approximate threshold value given by

$$
I_{\text {ave }}<U_{0} / \tau,
$$

where $U_{0}$ is the critical exposure energy to cure the photopolymer and $\tau$ is the photogenerated radical lifetime. For a typical photopolymer composition exhibiting trapping (photoinitiator concentration $0.005 \mathrm{wt} \%, U_{0} \approx 1 \mathrm{~J} \mathrm{~cm}^{-2}, \tau \approx 100$ $\mathrm{ms}$ ), the average intensity must be less than $10 \mathrm{~W} \mathrm{~cm}^{-2}$. Therefore, this optical nonlinearity is unique in that it occurs only for low average optical intensity. Intuitively, if intense illumination were used, a large number of radicals would be produced in a short time $t<\tau$ and the photopolymer would cure completely in the illuminated region, before the optical beam experienced an index change.

Standard negative photoresists are very similar to the photopolymers used here, indicating that this dramatic nonlinear optical response may play an important role in projection lithography. We propose two lithography processes

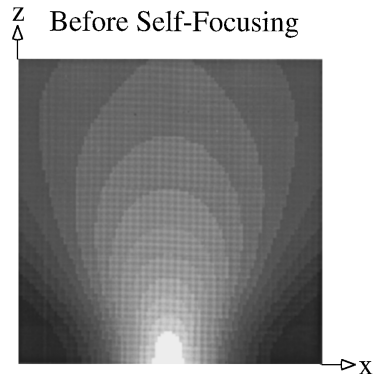

(a)

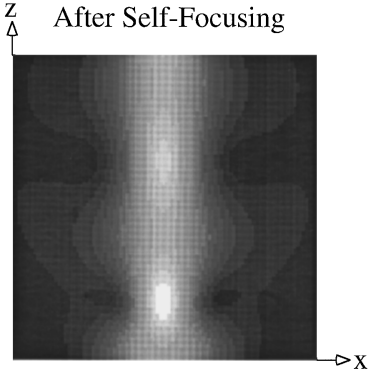

(b)
FIG. 3. Numerical simulations of self-focusing in resolution enhancement layer. Beam propagation before (a) and after (b) photopolymerization. Input beam radius is two wavelengths. Vertical scale 100 wavelengths, horizontal scale 10 wavelengths.

which take advantage of this effect. A standard positive photoresist may be overcoated with a resolution enhancement layer (REL) composed of a nonlinear photopolymer that selffocuses the light before developing the underlying photoresist [Fig. 2(a)]. A photobleachable dye may be placed between the REL and resist to ensure that the light-induced lens is induced before the underlying resist is exposed. The addition of this REL to optical lithography processes has the potential of reducing the feature size to half an exposure wavelength using existing steppers and photoresists. Alternately, self-trapping enables resists significantly thicker than the depth of focus to be patterned [Fig. 2(b)]. In this case the nonlinear optical property of the resist itself is used. These thick resists are used to produce, for instance, thicker interconnection layers which reduce ohmic losses.

We model the propagation of the beam through the photopolymer by the beam propagation method ${ }^{6}$ and observe propagation characteristics typical of self-focusing. A numerical simulation of the propagation of a single strip of light through a REL indicates that the linewidth may be reduced by more than a factor of 2 upon propagating through the nonlinear resist (Fig. 3). If the waveguiding is strong enough, the focused optical beam becomes trapped as it propagates, dramatically extending the depth of focus. Figure 3(a) illustrates the evolution of the beam through the homogeneous photopolymer before any index changes are induced. After a sequence of several short UV exposures, Fig. 3(b) exhibits self-focusing in addition to oscillatory self-

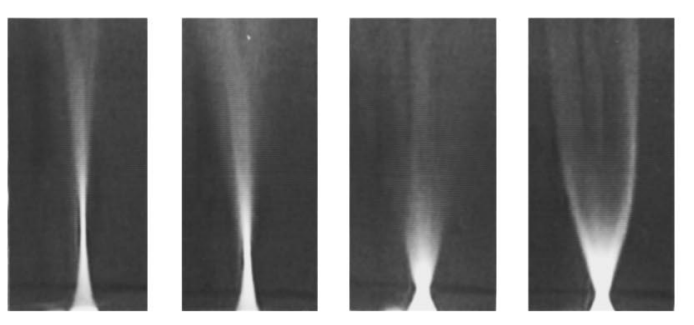

(a)

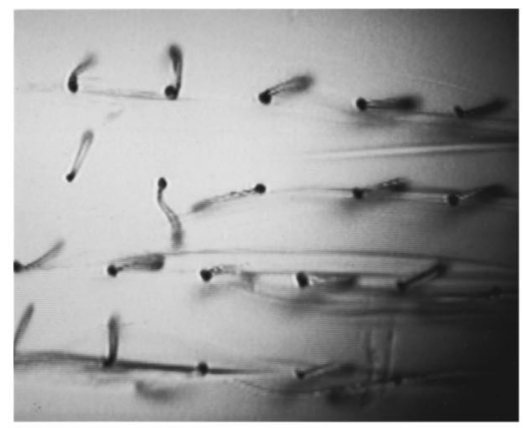

(b)

FIG. 4. (a) Experimental observation of self-focusing in photopolymer; input beam diameter $=100 \mu \mathrm{m}$. (b) Experimental observation of self-trapping in photopolymer; diameter of individual fibers $=10 \mu \mathrm{m}$ and length $=3 \mathrm{~mm}$. 

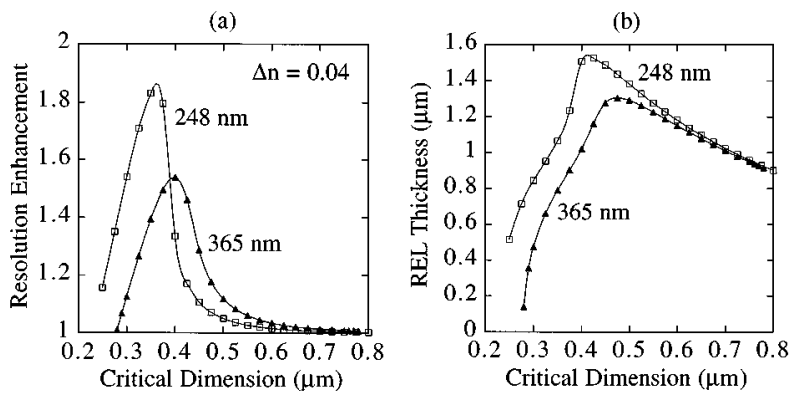

FIG. 5. (a) Theoretical enhancement in resolution at two standard wavelengths for photolithography $(248,365 \mathrm{~nm})$ as a function of linewidth. A maximum linewidth reduction of approximately 1.9 is predicted for $248 \mathrm{~nm}$ illumination. (b) Corresponding thicknesses of resolution enhancement layer.

trapping behavior. The waist of the beam is located at $z=0$, the input surface of the photopolymer. The beam width at the input is equal to two wavelengths. The horizontal scale is magnified by a factor of 10 compared to the vertical scale, to improve transverse resolution.

We have demonstrated self-focusing and self-trapping experimentally in a liquid diacrylate photopolymer. The input beam is a $1 \mathrm{~mW}, \mathrm{TEM}_{00}$ Gaussian beam at $325 \mathrm{~nm}$ with a $100 \mu \mathrm{m}$ beam diameter at the entrance to the photopolymer. The left-hand side of Fig. 4(a) illustrates the beam profile at $t=0$ inside the liquid photopolymer. Time increases from left to right. The right-hand side of Fig. 4(a) is taken after approximately $30 \mathrm{~s}$ of continuous illumination. The position of the beam waist moves closer to the input plane as photopolymerization proceeds, a manifestation of selffocusing. Figure 4(b) illustrates an array of polymer fibers of nearly constant $10 \mu \mathrm{m}$ diameter over a $3 \mathrm{~mm}$ length formed by self-trapping. In the absence of self-trapping, the crosssectional area of the fiber would have increased by a factor of 2 over a distance of $350 \mu \mathrm{m}$.

We approximate the self-focusing properties by modeling the index profile responsible for focusing a Gaussian input beam as a truncated quadratic. The focusing properties of this optical system under the paraxial approximation are then well known. ${ }^{7}$ For instance, we assume the waist of a one-dimensional Gaussian beam is incident on the top surface of a nonlinear photopolymer overcoating a standard photoresist [Fig. 2(a)]. The one-dimensional feature may represent an interconnection on an integrated circuit, for example. As this line propagates through the photopolymer, the linewidth is reduced. Figure 5(a) illustrates the corresponding resolution enhancement factors upon passing through the photopolymer at two common wavelengths for photolithography, 248 and $365 \mathrm{~nm}$. The resolution enhancement factor is defined as the ratio of the linewidth of the optical projection system to the linewidth after propagating through the nonlinear polymer. Figure 5(b) illustrates the corresponding thickness of the resolution enhancement layer which generates this focusing effect, for a light-induced index change of 0.04 . Note that the focusing properties depend strongly on the linewidth itself. Therefore, we expect that this resolution enhancement method is best suited for lithography patterns composed of regular features. Further work is presently being undertaken to refine these calculations for nonparaxial beam propagation.

There are several requirements on the photopolymer system to realize a practical REL. First, the optical intensity must be less than the intensity threshold dictated by the nonlinear response time of the polymer, which is typically in excess of a ms. Second, the sensitivity of the REL must be high enough that wafer throughput is not sacrificed. Third, the resolution of the REL must better than $0.3 \mu \mathrm{m}$. Fourth, the projected patterns must be composed of lines of predetermined width. Finally, the REL must be easily removed by a suitable solvent.

Of course, a typical photolithography mask is not composed simply of lines as our discussion has implicitly assumed. However, for certain critical patterning stages composed of fairly regular structures, this technique may be of value. On the other hand, deleterious nonlinear optical effects such as self-defocusing and distortion arising from the same phenomenon should be avoided. Positive photoresists are expected to exhibit a smaller change in the index of refraction compared to negative photoresists. In some situations the nonlinearities should be avoided, in other cases embraced. The nonlinearities are avoided by selecting photoresists with exposure thresholds significantly less than the intensity of the UV source. As linewidths continue to shrink, these nonlinear optical effects are expected to become more pronounced and should be included in future photoresist profile modeling.

This work was supported by ARPA's nonlinear optics (DSO) and lithography (MTO) programs.

${ }^{1}$ B. F. Griffing and P. R. West, Solid State Technol. 1985, May, 152.

${ }^{2}$ S. V. Babu, E. Barouch, and B. Bradie, J. Vac. Sci. Technol. B 6, 564 (1988).

${ }^{3}$ D. C. Hofer, C. G. Wilson, A. R. Neureuther, and M. Hakey, Proc. SPIE 334, 196 (1982)

${ }^{4}$ A. S. Kewitsch and A. Yariv, Opt. Lett. 21, 24 (1996).

${ }^{5}$ W. J. Tomlinson and E. A. Chandross, in Advances in Photochemistry, edited by J. J. N. Pitts, G. S. Hammond, and K. Gollnick (Wiley, New York, 1985), Vol. 12, pp. 201-281.

${ }^{6}$ Y. Chung and N. Dagli, IEEE J. Quantum Electron. 26, 1335 (1990).

${ }^{7}$ A. Yariv, Quantum Electronics, 3rd ed. (Wiley, New York, 1989). 